\title{
Novel assay for efficient iron release from ferritin
}

\begin{abstract}
Ferritin plays an important role in iron detoxification and iron storage by converting soluble iron(II) to insoluble iron(III) inside the protein cage. Mechanism of in vitro ferritin iron release occurs more readily under reducing conditions, whereby reducing agents may enter into the ferritin cage through the protein pores to convert insoluble iron(III) into soluble iron(II), after accepting an electron from reducing agents. The soluble iron(II) then liberates outside the protein cage and forms coloured and stable complexes in the presence of chromophore, an iron(II) chelating agent, to be quantified by spectrophotometry. However, iron(II) is readily converted back to iron(III) in the presence of oxygen. Thus, oxygen scavenger compounds were used to remove any dissolved oxygen. Studies suggested that low concentration of chaotropic agents regulate the protein pore opening and result in protein unfolding that is therefore accessible by reducing agents more effectively. According to in vivo studies, iron exit from ferritin for recycling or meeting the cell's need via lysosomal and/or proteasomal pathways. We designed an assay to determine the total iron available inside the ferritin by first using the commercially available iron(II) or iron(III) compounds as a substrate to optimize the concentration of reactants involved. Apart from chromophore and reducing agents, oxygen scavenger compounds, chaotropic agents and enzymes were added in order to induce ferritin iron release effectively. Linear calibration curve of the iron(II)chromophore complex were obtained and was used as a reference to correlate absorbance readings to concentration of iron level. Reducing agents was able to reduce the iron(III) to iron(II) and oxygen scavenger A seems to be more effective in removing any dissolved oxygen to avoid oxidation of both the reducing agents and iron(III). Taken altogether, this experiment setup will be beneficial for the improvement of efficiency in quantifying the total iron level inside the ferritin.
\end{abstract}

Keyword: Ferritin; Iron; Chaotropic agents; Low concentrations; Oxygen scavengers 